\title{
Spin Density Matrix Elements in Exclusive Production of Omega Mesons at HERMES
}

\author{
Hrachya Marukyan \\ (on behalf of the HERMES collaboration) \\ A. Alikhanian National Science Laboratory \\ (Yerevan Physics Institute) \\ Yerevan, 0036, Armenia \\ marukyan@mail.desy.de
}

Published 29 February 2016

\begin{abstract}
Exclusive electroproduction of $\omega$ mesons on unpolarized hydrogen and deuterium targets is studied at HERMES in the kinematic region of $Q^{2}>1.0 \mathrm{GeV}^{2}, 3.0 \mathrm{GeV}<\mathrm{W}<$ $6.3 \mathrm{GeV}$, and $-t^{\prime}<0.2 \mathrm{GeV}^{2}$. The data were accumulated during the 1996-2007 running period using the $27.6 \mathrm{GeV}$ longitudinally polarized electron or positron beams at HERA. The determination of the virtual-photon longitudinal-to-transverse cross-section ratio shows that a considerable part of the cross section arises from transversely polarized photons. Spin density matrix elements are derived and presented in projections of $Q^{2}$ or $-t^{\prime}$. Violation of s-channel helicity conservation is observed for some of these elements. A sizable contribution from unnatural-parity-exchange amplitudes is found and the phase shift between those amplitudes that describe transverse $\omega$ production by longitudinal and transverse virtual photons is determined for the first time. Good agreement is found between the HERMES proton data and results of a pQCD-inspired phenomenological model that includes pion-pole contributions.
\end{abstract}

Keywords: Exclusive reactions; vector mesons; omega meson production.

PACS numbers: 13.60.Le, 13.88+e, 13.20.Jf, 14.20.Dh, 12.38.Qk

\section{Spin Density Matrix Elements}

Exclusive electroproduction of vector mesons on nucleons provide a rich source of information on the structure of the nucleon and the meson production mechanism. The angular distribution of vector mesons in this reactions depends on Spin Density Matrix Elements (SDMEs), which describe the final spin state of produced mesons. SDMEs are expressed by the helicity amplitudes describing the transition of longitudinal or transverse virtual photons to the vector meson with given helicity.

This is an Open Access article published by World Scientific Publishing Company. It is distributed under the terms of the Creative Commons Attribution 3.0 (CC-BY) License. Further distribution of this work is permitted, provided the original work is properly cited. 
HERMES has measured the values of 23 SDMEs in exclusive electroproduction of $\omega$ mesons on unpolarized hydrogen and deuterium targets with longitudinally polarized electron or positron beams at HERA. ${ }^{1}$ They are determined from the process $e+p(d) \rightarrow e^{\prime}+p^{\prime}\left(p^{\prime} n^{\prime}\right)+\omega \rightarrow\left(\pi^{+} \pi^{-} \pi^{0}(\rightarrow 2 \gamma)\right)$ by fitting the 3 -dimensional angular distribution, which depends on the angle between the lepton scattering and production planes and the polar and azimuthal angles of a unit vector normal to the decay plane in the rest frame of $\omega$, to the experimental angular distribution of decay pions. Fitting is done with the unbinned maximum likelihood method (for details see Ref. ${ }^{1}$ ).

\section{Results}

The values of SDMEs of the $\omega$ - meson for the entire HERMES kinematic region $\left(\left\langle Q^{2}\right\rangle=2.42 \mathrm{GeV}^{2},\langle W\rangle=4.8 \mathrm{GeV}\right.$ and $\left.\left\langle-t^{\prime}\right\rangle=0.08 \mathrm{GeV}^{2}\right)$, where $Q^{2}$ represents the negative squared virtual-photon four-momentum, $W$ the invariant mass of the photon-nucleon system and $t^{\prime}$ the difference between the Mandelstam variable $t$ and its kinematically allowed smallest value, are presented in Fig. 1. As can be seen from this figure, the SDMEs are divided into five classes, corresponding to different helicity transitions. Class A corresponds to the transition of longitudinal virtual photons to longitudinal mesons $\gamma_{L}^{\star} \rightarrow V_{L}$, and of transverse virtual photons to transverse mesons $\gamma_{T}^{\star} \rightarrow V_{T}$. Class B corresponds to the interference of these two transitions. Class $\mathrm{C}$ corresponds to the $\gamma_{T}^{\star} \rightarrow V_{L}$ transition, class D to the $\gamma_{L}^{\star} \rightarrow V_{T}$ transition, and class E to the $\gamma_{T}^{\star} \rightarrow V_{-T}$ transition. For comparison, the values of SDMEs in exclusive electroproduction of the $\rho^{0}$ meson $^{2}$ measured at HERMES are also plotted in Fig. 1.

The SDMEs for the hydrogen and deuterium data are found to be consistent within their quadratically combined total uncertainties. The eight polarized SDMEs presented in the shaded areas are larger compared to the unpolarized SDMEs due to the lepton beam polarization, which is smaller than unity. The SDMEs $r_{1-1}^{1}$ and $\operatorname{Im} r_{1-1}^{2}$ of class A have opposite signs for $\omega$ and $\rho^{0}$, which suggests a large Unnatural Parity Exchange (UPE) contribution in exclusive $\omega$ meson production.

In the case of so-called S-Channel Helicity Conservation (SCHC), i.e., the dominance of diagonal transitions, the seven SDMEs of class A and B $\left(r_{00}^{04}, r_{1-1}^{1}, \operatorname{Im} r_{1-1}^{2}\right.$, $\operatorname{Re} r_{10}^{5}, \operatorname{Im} r_{10}^{6}, \operatorname{Im} r_{10}^{7}$ and $\left.\operatorname{Re} r_{10}^{8}\right)$ are not restricted to be zero. Indeed, as can be seen in Fig. 1, they are mostly non-zero. In addition, six of these SDMEs obey the relations $r_{1-1}^{1}=-\operatorname{Im} r_{1-1}^{2}, \operatorname{Re} r_{10}^{5}=-\operatorname{Im} r_{10}^{6}$ and $\operatorname{Im} r_{10}^{7}=\operatorname{Re} r_{10}^{8}$, which hold mostly within one standard deviation.

As an example, the $Q^{2}$ and $-t^{\prime}$ dependence of class-A SDMEs for exclusive $\omega$ meson electroproduction is presented in Fig. 2 for proton and deutron, respectively. The measured values are in good agreement with the pQCD-inspired phenomenological "GK model" calculations, ${ }^{3}$ in which the unnatural-parity contributions due to pion exchange are included. It was based on a model which describes the deeply virtual Compton scattering rather well. ${ }^{4}$ 


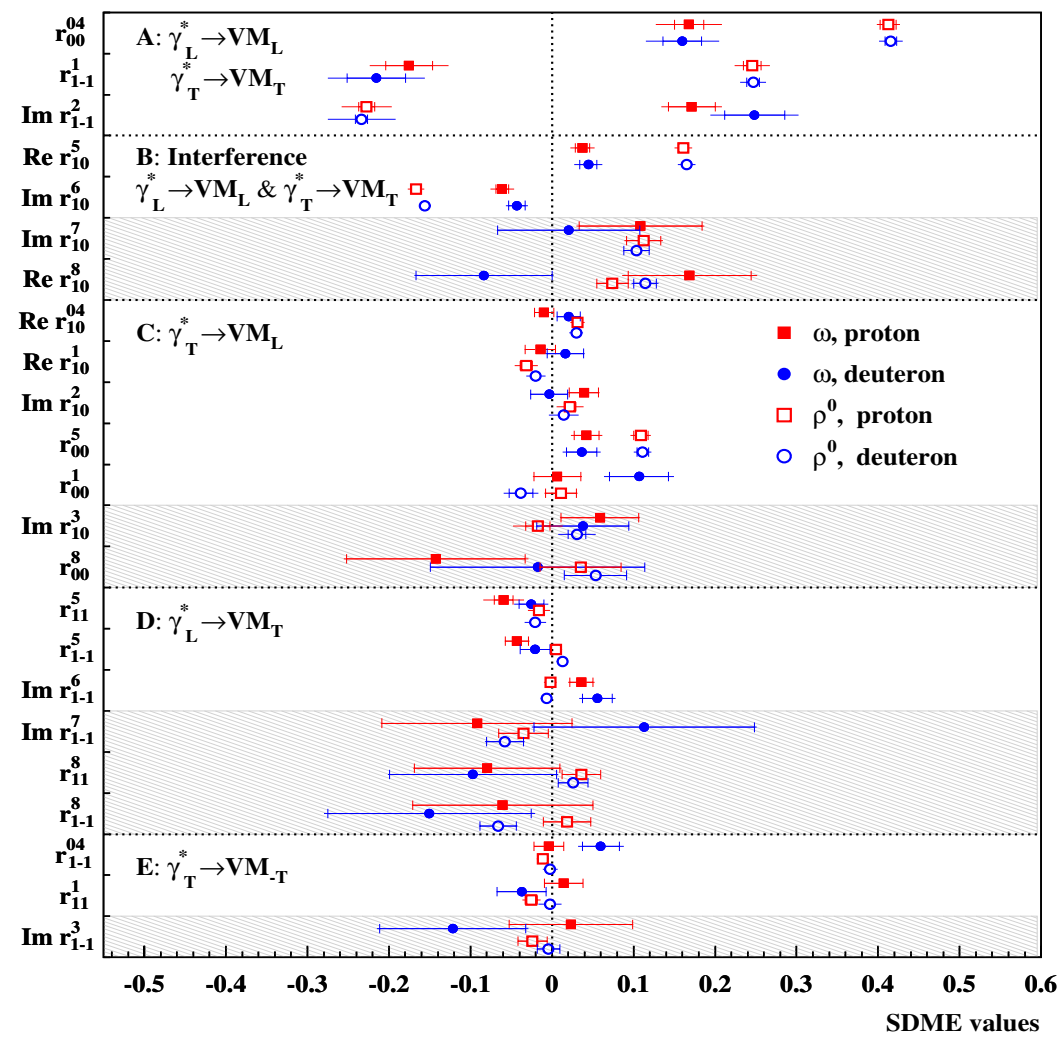

Fig. 1. The SDMEs in exclusive $\omega$ and $\rho^{0}$ electroproduction at HERMES: proton (circles) and deuteron (squares) in the entire HERMES kinematic region. The inner error bars represent the statistical uncertainties, while the outer error bars indicate the statistical and systematic uncertainties added in quadrature. SDMEs measured with unpolarized (polarized) beam are displayed in the unshaded (shaded) areas.

The existence of UPE in $\omega$ production was tested directly through a non-zero result for the quantities $u_{1}, u_{2}$ and $u_{3}$, which are presented respectively by linear combinations of SDMEs, such as $u_{1}=1-r_{00}^{04}+2 r_{1-1}^{04}-2 r_{11}^{1}-r_{1-1}^{1}, u_{2}=r_{11}^{5}+r_{1-1}^{1}$ and $u_{3}=r_{11}^{8}+r_{1-1}^{8}$.

In Fig. 3, the $Q^{2}$ and $-t^{\prime}$ dependence of $u_{1}$ for the proton and deuteron is presented. One can see that $u_{1}$ is larger than unity, implying large contributions from UPE transitions. This suggests that at HERMES energies the quark-exchanges mechanism or the $\pi_{0}$-exchange in Regge phenomenology play a significant role in $\omega$ meson electroproduction. The results are in good agreement with GK model calculations. ${ }^{3}$ For exclusive $\omega$ meson production $u_{2}$ for the proton is clearly non 

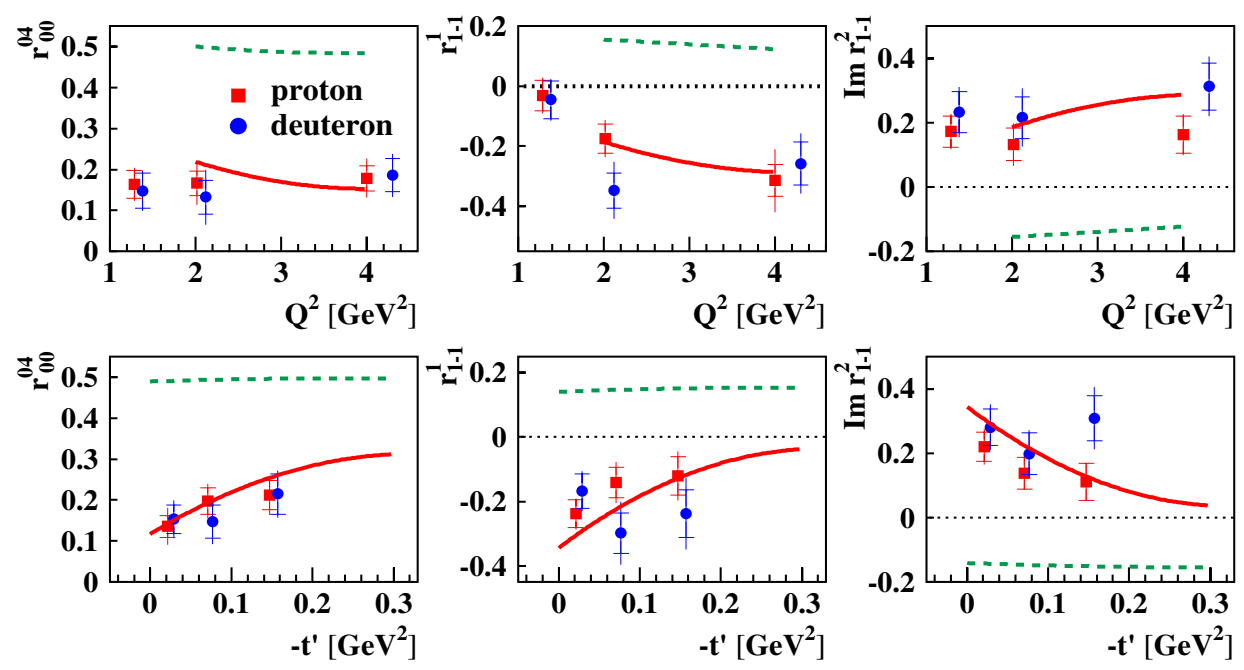

Fig. 2. The $Q^{2}$ and $-t^{\prime}$ dependence of class-A SDMEs. Proton (deutron) data are denoted by squares (circles). The inner error bars represent the statistical uncertainties, while the outer error bars indicate the statistical and systematic uncertainties added in quadrature. The proton data are compared to calculations of the GK model with (solid curve) and without (dashed curve) pion-pole contributions.
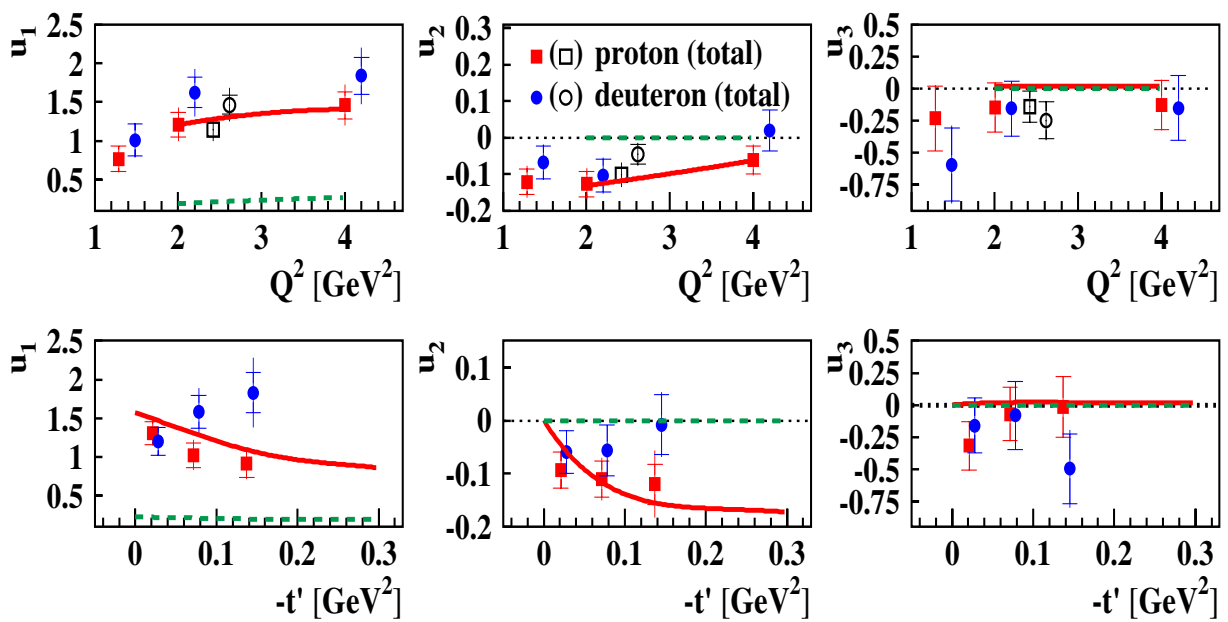

Fig. 3. The $Q^{2}$ and $-t^{\prime}$ dependence of $u_{1}, u_{2}$ and $u_{3}$ for exclusive $\omega$ production for hydrogen (squares) and deuterium (circles). The open symbols represent the values for entire kinematic region. Otherwise as for Fig. 2. 

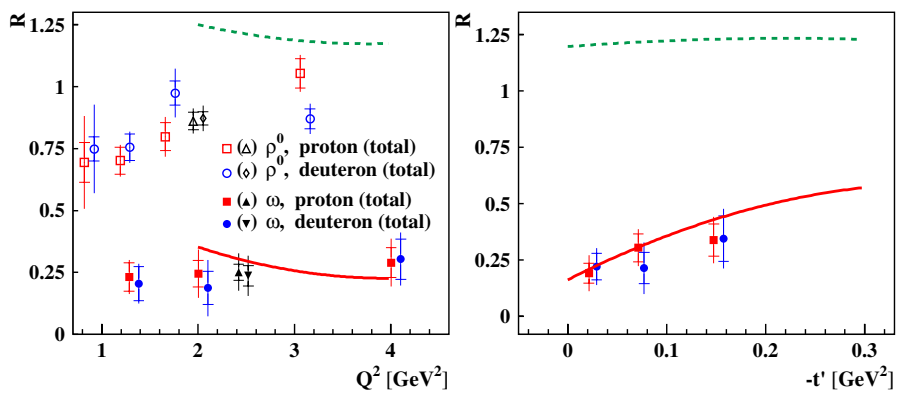

Fig. 4. The $Q^{2}$ (left) and $-t^{\prime}$ (right) dependence of the longitudinal-to-transverse virtual-photon cross-section ratio for exclusive $\omega$ and $\rho^{0}$ electroproduction at HERMES. The symbols in parenthesis represent the value of $R$ in the entire kinematic region. Otherwise as for Fig. 2.

zero, and conclusion on $u_{3}$ suffers from considerable experimental uncertainties, while for $\rho^{0}$ meson production both $u_{2}$ and $u_{3}$ are compatible with zero. ${ }^{2}$

For exclusive $\omega$ meson production, the $Q^{2}$ and $-t^{\prime}$ dependence of the longitudinal-to-transverse virtual-photon differential cross-section ratio $R$ determined from the measured SDME $r_{00}^{04}$ is shown in Fig. 4. It is found that the values of $R$ for the proton are compatible with those obtained for the deuteron. This ratio is about four times smaller than for the $\rho^{0}$ meson, ${ }^{2}$ which is shown in the left panel of Fig. 4. The proton data are well described by the GK model with the inclusion of pion-pole ${ }^{3}$ contributions, and thus, fully account for the unnatural parity contribution to $R$.

The $Q^{2}$ and $-t^{\prime}$ dependence of the UPE to Natural Parity Exchange (NPE) asymmetry of the transverse differential cross-section $P$ for the exclusive $\omega$
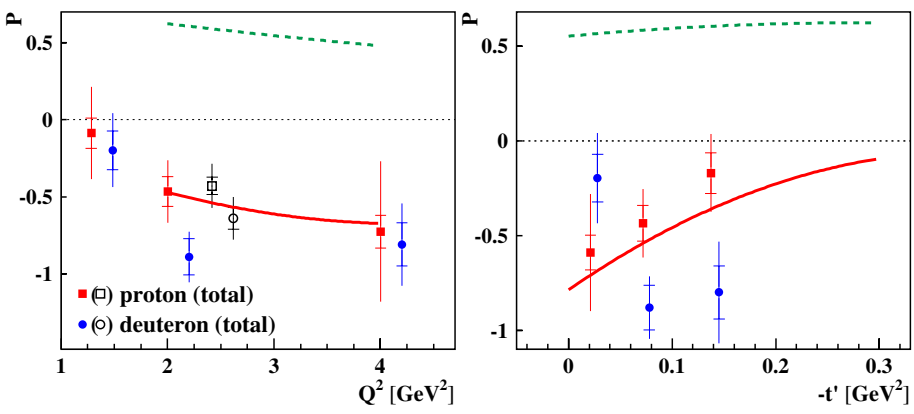

Fig. 5. The $Q^{2}$ and $-t^{\prime}$ dependence of the asymmetry of unnatural parity to natural parity exchange $P$ of the transverse differential cross-section for exclusive $\omega$ electroproduction at HERMES. The open symbols represent the values over entire kinematic region. Otherwise as for Fig. 2. 

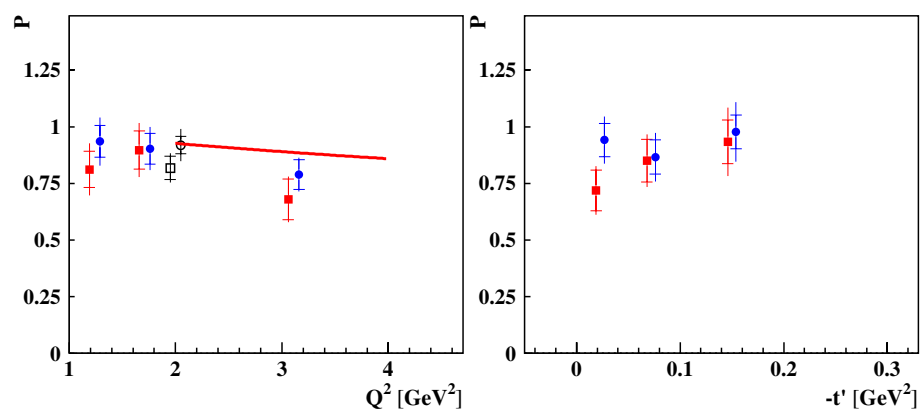

Fig. 6. The $Q^{2}$ and $-t^{\prime}$ dependence of the asymmetry of unnatural parity to natural parity exchange $P$ of the transverse differential cross-section for exclusive $\rho^{0}$ electroproduction at HERMES. The open symbols represent the values over entire kinematic region. Otherwise as for Fig. 2.

production is shown in Fig. 5. Asymmetry values significantly different from unity are obtained for both the proton and deuteron, which means that a large part of transverse cross section is due to the UPE. The calculations of the GK model ${ }^{3}$ with pion-pole contributions show very good agreement with the data both in shape and magnitude. For comparison, in Fig. 6 the same asymmetry vs. $Q^{2}$ and $-t^{\prime}$ measured at HERMES for exclusive $\rho^{0}$ meson $^{2}$ is plotted. They are calculated from extracted values of the SDMES $r_{00}^{04}, r_{0}^{1-1}$ and $r_{1}^{00}$ given in Ref.. ${ }^{2}$ There is good agreement of data with the GK model including the pion-pole contributions (see left panel). Note that the calculations without pion-pole contributions are not shown in Fig. 6 for $\rho^{0}$. The difference between the two curves with and without pion-pole contributions is not significant in this case, which means that the pion-pole gives big contributions for exclusively produced $\omega$ mesons, but not for the $\rho^{0}$ (see Ref. ${ }^{3}$ ).

\section{Acknowledgments}

We are grateful to S.V. Goloskokov and P. Kroll providing the results of their calculations. I would also like to thank the organizers for their support and for a very interesting conference.

\section{References}

1. A. Airapetian et al, Eur. Phys. J. C 74, 3110 (2014).

2. A. Airapetian et al, Eur. Phys. J. C 62, 659 (2009).

3. S.V. Goloskokov and P. Kroll, Eur. Phys. J. A 50, 146 (2014).

4. P. Kroll, H. Moutarde and F. Sabatié Eur. Phys. J. C 73, 2278 (2013). 